\title{
O ÍNDICE DE
}

\section{DESENVOLVIMENTO}
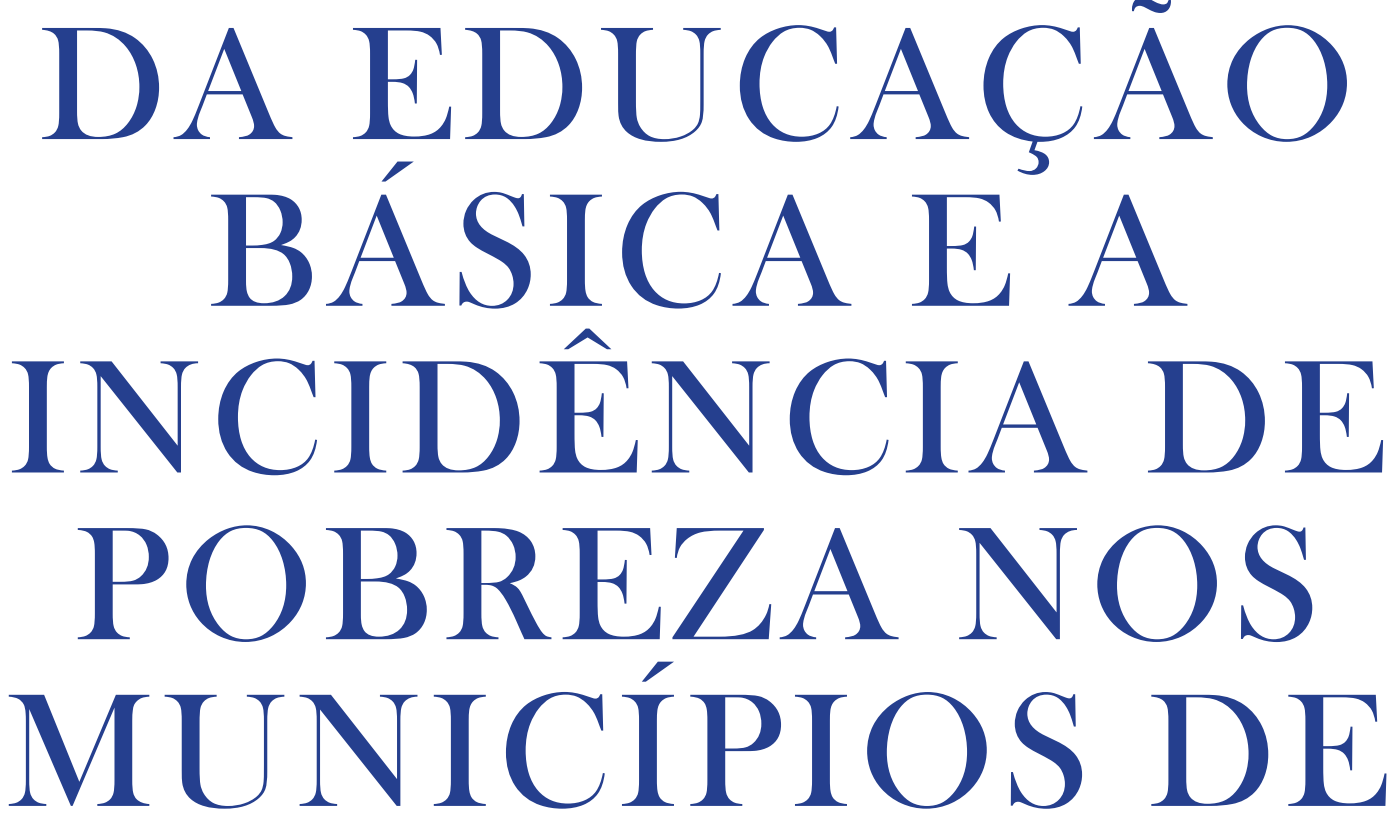

PEQUENO PORTE
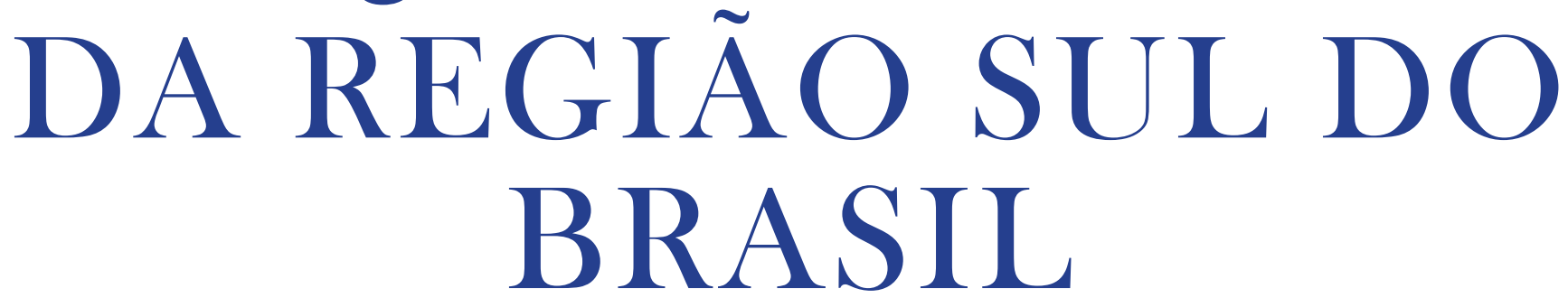

THE DEVELOPMENT INDEX OF BASIC EDUCATION AND POVERTY LEVELS IN SMALL MUNICIPALITIES OF SOUTHERN BRAZIL

EL ÍNDICE DE DESARROLLO DE LA EDUCACIÓN BÁSICA Y LA INCIDENCIA DE LA POBREZA EN LAS PEQUEÑAS CIUDADES DEL SUR DE BRASIL

Valdecir Soligo ${ }^{1}$

${ }^{1}$ Doutor em Educação pela UNISINOS. Docente no Curso de Pedagogia da Universidade Estadual do Oeste do Paraná (UNIOESTE) - Cascavel - PR - Brasil.

Resumo: A interferência das condições sociais no rendimento escolar não é novidade para a ciência. $O$ que motiva esta investigação é a insuficiência de estudos quantitativos com os dados disponibilizados pelo IBGE 
e INEP referentes à educação e às condições sociais da população brasileira como ferramentas de planejamento da gestão pública. O objetivo foi analisar em que medida e intensidade as condições sociais interferem no rendimento escolar. Para tanto foi utilizado regressão linear entre as variáveis: Incidência de Pobreza e Valor médio de IDEB para municípios com menos de 10 mil habitantes e que apresentam ambas as variáveis na Região Sul do Brasil. Como resultado, os valores indicam que há uma relação inversamente proporcional, demonstrando que, conforme aumentam os valores da Incidência de Pobreza, diminuem os valores Médios do IDEB. Outro aspecto revelado na pesquisa é a diferença entre os resultados da Região Sul e da análise de cada Unidade Federativa que compõem a Região em separado, indicando fragilidade das análises estatísticas de grande proporção.

Palavras Chave: Avaliação; Pobreza; Rendimento Escolar.

Abstract: The influence of social conditions on school performance is not new to science. This research was prompted by the lack of quantitative studies with data released by the IBGE (Brazilian Institute of Geography and Statistics) and INEP (Anísio Teixeira National Institute of Educational Studies and Research) relating to education and the social conditions of the Brazilian population as a public management planning tool. The objective was to analyze the extent to which social conditions affect school performance. For this, we used linear regression between the variables Poverty Incidence and Average value of IDEB (Basic Education Development Index), for municipalities in southern Brazil with less than 10,000 inhabitants and that present both variables. As a result, the values indicate 
that there is an inverse relationship, showing that as the values for Incidence of Poverty increased, the Mean values of the IDEB decreased. Another aspect revealed in the survey is the difference between the results for the South region and the analysis of each Federal Unit that comprises the Region separately, indicating a weakness in large proportion statistical analyses.

Keywords: Evaluation; Poverty; Educational Achievement.

Resumen: La interferencia de las condiciones sociales en el rendimiento escolar no es nueva para la ciencia. Lo que nos motiva en esta investigación es la falta de estudios cuantitativos con los datos publicados por el IBGE y el INEP relacionados a la educación y a las condiciones sociales de la población brasileña como herramientas de planificación de la gestión pública. El objetivo fue analizar en qué medida e intensidad las condiciones sociales interfieren en el rendimiento escolar. Para ello se utilizó la regresión lineal entre las variables de incidencia de la pobreza y el valor promedio del IDEB para los municipios con menos de 10.000 habitantes y que presentan ambas variables en la Región Sur de Brasil. Como resultado, los valores indican que existe una relación inversamente proporcional, lo que demuestra que conforme aumentan los valores de incidencia de la pobreza, disminuyen los valores medios del IDEB. Otro aspecto revelado en la encuesta es la diferencia entre los resultados de la Región Sur y el análisis de cada Unidad de la Federación que compone la Región por separado, indicando la fragilidad de los análisis estadísticos de gran proporción.

Palabras clave: Evaluación; Pobreza; Rendimiento escolar. 
ste estudo parte da preocupação como pesquisador em torno do volume de informações disponibilizadas por agências produtoras de estatística, referentes às condições sociais e educacionais da população brasileira e seu uso por gestores educacionais dos municípios de pequeno porte da Região Sul do Brasil. Como resultado desta inquietação, desenvolveu-se esta pesquisa de doutoramento, na qual são analisados indicadores sociais e suas relações com o rendimento escolar aferido pelas avaliações em larga escala.

Para a discussão neste artigo, foram selecionadas as variáveis: a Incidência de Pobreza e o Índice de Desenvolvimento da Educação Básica (IDEB). O objetivo é analisar as relações entre esses indicadores que compõem um conjunto de informações disponibilizadas nos sites do Instituto Brasileiro de Geografia e Estatístico (IBGE), e Instituto Nacional de Estudos e Pesquisas Educacionais Anísio Teixeira (INEP). Os indicadores sociais aqui analisados trazem informações sobre o percentual de pobres no interior de cada município e entre os municípios da região objeto e o rendimento escolar. $\mathrm{O}$ indicador Incidência de Pobreza foi analisado em comparação direta com a média do IDEB de cada município, por Estado e no conjunto da Região Sul do Brasil. Os resultados serão apresentados em momento oportuno no decorrer deste texto, antes se faz necessário retomar alguns apontamentos teórico-metodológicos sobre a temática.

Os estudos sobre rendimento escolar, encontrados em periódicos e no Banco de Teses da CAPES, apresentam multiplicidade de foco e métodos. Isso se verifica devido à crescente preocupação com a qualidade da Educação e com os sistemas de avaliação em larga escala nos últimos anos.

Uma das principais preocupações da sociedade brasileira vem sendo o acesso à Educação Básica. Desde as reformas educativas, iniciadas no fim dos anos de 1980 e que vêm sendo perseguidas até os dias atuais, essa é uma problemática recorrente na agenda de políticas educacionais. Constitui-se em diretrizes e leis que buscam regular a oferta e a qualidade, resultando, por exemplo, na obrigatoriedade da matrícula e de permanência de crianças e jovens, entre seis e quatorze anos de idade, em escolas regulares.

A Lei de Diretrizes e Bases (LDB), aprovada em 1996, define e regulariza o sistema de educação brasileiro, com base nos princípios presentes na Constituição 
Federal de 1988 (CF). Ambas as leis são resultado de amplo debate acadêmico e de ajustes políticos que visavam melhorar a qualidade da Educação, tanto em acesso e permanência, quanto em qualidade de ensino. Os efeitos podem ser percebidos com maior clareza, quando considerados os percentuais de crianças com idade entre seis e quatorze anos matriculadas na Educação Básica. Com 97\% das crianças em idade escolar na escola, a universalização do Ensino Fundamental deixa de ser o grande entrave da educação brasileira. Com o problema do acesso à escola no Ensino Fundamental praticamente solucionado, retoma-se a qualidade do ensino oferecido como a grande problemática da Educação (Soares, 2004).

Na expectativa de garantir o padrão de qualidade, a LDB/96 incumbiu à União o dever de assegurar o processo nacional de avaliação do rendimento escolar, que, a partir do final da década de 1980, havia começado a ser implementado no Brasil por meio dos Sistemas de Avaliações em Larga Escala.

Essas avaliações, em discurso oficial, são apresentadas como uma forma de verificar a qualidade da Educação. Na prática, mensuram parte das habilidades e das competências de leitura em Língua Portuguesa e resolução de problemas matemáticos cruzados com as informações do Censo Escolar sobre fluxo. $O$ resultado desse processo gera o Índice de Desenvolvimento da Educação Básica (IDEB).

No debate sobre qualidade da Educação, o IDEB vem sendo apresentado, pela imprensa, como sinônimo de qualidade. Esse modelo de divulgação desconsidera os processos e as diferenças socioculturais e econômicas do país, possibilitando a construção de ranking.

A qualidade, ainda que não seja um tema novo, atualmente toma dimensões significativas nas políticas educacionais e nos debates acadêmicos, revelando inúmeros fatores que podem ser relacionados com a melhoria ou com a piora da qualidade mensurável em testes padronizados.

Nesse aspecto, o aumento de alunos na Educação Básica pode ter favorecido a queda do desempenho geral dos alunos, em um passado não muito distante, também no Brasil, devido, principalmente, ao modelo de educação de massa com baixo custo, adotado para o país. Souza (2004) alerta, entretanto, para o fato de que é o discurso oficial que justifica a tendência de queda, a partir da expansão das matrículas ocorridas nos anos anteriores às avaliações do Sistema 
de Avaliação da Educação Básica (SAEB). Segundo o autor, a evolução negativa no desempenho dos estudantes brasileiros, entre 1995 e 1999, deveria ser interpretada como positiva, pois:

Os novos integrantes do sistema estão muito melhor do que estavam antes de ingressarem na escola, pois estão aprendendo. Seu desempenho inicial, porém, é inferior em relação aos demais alunos que já estavam na escola e que provêm de famílias mais educadas. Como conseqüência, a média do novo conjunto de estudantes é menor do que a existente antes da incorporação maciça dos novos alunos (Souza, 2004, p.19).

Quando se afirma que a entrada de um grande contingente de alunos, que até então não tinham acesso à escolaridade, é a causadora da queda de desempenho geral, acaba-se por responsabilizar este público pelo desempenho fraco do SAEB. Por outro lado, é com esse público que se verifica quanto é válida a afirmação de que as condições sociais e culturais implicam o rendimento escolar.

Outros fatores sociais, econômicos e culturais podem estar relacionados ao desempenho escolar. Ainda que os governos, por meio de programas, como o Bolsa Família, Merenda Escolar, Livro Didático, Transporte Escolar, etc., busquem garantir a permanência das crianças nas escolas, o problema da repetência continua assolando a educação brasileira. A taxa de distorção idade/série para $4^{a}$ Série $/ 5^{\circ}$ Ano média nacional, em 2009, era de $23 \%$, o que indica parte desta problemática.

A relação entre rendimento escolar e indicadores sociais, econômicos e educacionais destaca-se no debate sobre qualidade da Educação como um fator preponderante, pois revela as disparidades sociais e econômicas entre os municípios de pequeno porte, bem como das populações de cada município. Esse fator implica a ineficiência do sistema em oferecer ensino de qualidade, refletindo na reprovação, distorção idade série, analfabetismo funcional, abandono e baixos índices na própria avaliação do sistema.

As pesquisas sobre o desempenho escolar no Brasil levam em consideração uma série de fatores do aluno, da escola, dos professores, das famílias, etc. Ainda que, na atualidade, o destaque esteja nas avaliações em larga escala (Prova Brasil, IDEB, entre outros), que consideram parte de aspectos mensuráveis de leitura em Língua Portuguesa e resolução de Problemas Matemáticos, estudos como os de Soares \& Mambrini (2001); Andrade \& Laros (2007), Barbosa \& Fernandes (2001), entre outros, debatem inúmeros fatores. Nesse aspecto, o nível socioeconômico 1 Os possíveis significados desses dados não compõem o objeto de estudo desta pesquisa. 
familiar e o conhecimento prévio do aluno são exemplos de variáveis que podem afetar o desempenho.

O baixo rendimento dos alunos do Ensino Fundamental no Brasil, em muitos casos, é atribuído às condições socioeconômicas dos alunos. A literatura aponta para o fato de que diversos fatores determinam o desempenho escolar, dentre eles: qualidade do professor, nível socioeconômico da família e da escola que o aluno frequenta; escolaridade dos pais; infraestrutura das escolas; número de horas-aula; idade de entrada no sistema escolar; cor; atraso escolar; reprovação; presença de computadores em casa, etc. (Soares, 2004; Laros, 2001; Del Porto; Ferreira, 2007).

No âmbito mundial, o tema qualidade na Educação ganhou relevância quando os Estados Unidos realizaram, em 1966, um grande levantamento com 645 mil alunos, com objetivo de verificar o nível de conhecimento dos mesmos. O momento político que os americanos vivenciavam era de resgate dos direitos civis dos negros. A conclusão do relatório foi que o desempenho diferente dos alunos estava relacionado mais com a questão socioeconômica do que com as questões de dentro da escola (Coelho, 2008).

Considera-se esse relatório um marco na Educação, principalmente em se tratando de estatística educacional, pois, por conta da sua ampla divulgação, os países, inclusive o Brasil, iniciaram estudos sobre os fatores influenciadores no desempenho.

A evidência de que o nível socioeconômico influencia no desempenho do aluno é confirmada em estudos como o de Soares (2004). Por meio de dados do SAEB 2001, Soares considerou a desigualdade socioeconômica a principal variável que afeta o desempenho dos alunos e construiu um indicador socioeconômico para verificar a premissa.

Soares (2004), utilizando os resultados do SAEB 2001, constatou uma relação entre proficiência e nível socioeconômico, concluindo que a qualidade de ensino não se distribui de forma igualitária em todos os estratos populacionais. Para melhoria da Educação, o autor conclui que é necessário que haja a diminuição do impacto da posição social no sucesso escolar.

Para Soares (2004), no Brasil, existe uma dependência entre proficiência e nível socioeconômico. Os menos favorecidos, em sua maioria, possuem os piores desempenhos. Nesses termos, o autor elege alguns fatores que podem diminuir 
essa dependência. São eles: Políticas sociais, Políticas escolares e Contribuição da família.

Alves (2009) analisa a relação entre desempenho escolar e políticas educacionais nas capitais brasileiras por meio de duas medidas de desempenho escolar: alunos da $4^{a}$ série do Ensino Fundamental em idades distintas e gerações de crianças de 10 anos. Para isso utilizaram-se, dentre outras fontes, os dados do SAEB. No modelo estimado pela autora, o processo envolvendo escolha e eleição de diretores teve efeito significativo, ou seja, quando a escolha de diretores se dá de uma forma democrática, o desempenho dos alunos tende a aumentar em média 6,31 pontos, na escala do SAEB, em relação ao desempenho de alunos das redes que não adotam processos eletivos para escolha de diretores.

O desempenho em gerações (idade) foi discutido no texto de Fernandes (2007), que considera a proficiência média da geração, como mais adequada para monitorar o sistema de ensino (Brasil, Estados e Municípios). Del Porto e Ferreira (2007) buscaram associar fatores socioeconômicos e culturais com o desempenho dos alunos no PISA no Brasil e em mais três países (México, Espanha e Portugal). Esse estudo procurou detectar como as variáveis referentes aos capitais cultural, social e econômico afetam o desempenho dos estudantes em cada um desses países.

O modelo de regressão, aplicado por Del Porto e Ferreira (2007), indica a presença de fatores específicos nos países. No Brasil, as variáveis que explicam $30 \%$ da variância do desempenho em Matemática, de acordo com dados do PISA (2003), são os bens relacionados à cultura clássica em casa, Índice PISA socioeconômico internacional de status ocupacional do pai e da mãe ${ }^{2}$, computador e internet em casa, recursos domésticos educacionais e escolaridade dos pais.

Outros fatores que podem influenciar o desempenho: atraso escolar; comparação dos alunos com os colegas; recursos culturais de que o aluno dispõe em casa; gosto por estudar determinada disciplina; realização do dever de casa; relação da família do aluno com a escola; o fato de que o aluno trabalha (Andrade; Laros, 2007).

O levantamento bibliográfico vai além das pesquisas citadas anteriormente; entretanto, ainda que com grande variabilidade de fonte e métodos, é possível

2 O Índice Pisa socioeconômico internacional de status ocupacional é resultante das respostas dos estudantes sobre ocupação parental. O índice captou os atributos das ocupações que convertem a educação parental em renda. Derivou-se da escala ótima de grupos de ocupações para maximizar (Ganzeboom et al., 1992). 
perceber que a literatura aponta para a existência de fatores intraescolares e extraescolares de ordem social, econômica, cultural e educacional. Nestes termos, a desigualdade socioeconômica é uma das principais variáveis que afetam o desempenho dos alunos em testes padronizados segundo Soares (2004). Del Porto e Ferreira (2007) também percebem tal relação, da mesma forma que Alves (2008) e Gatti e Barreto (2009). Estes estudos, dentre outros, buscam identificar que fatores podem ser relacionados com o desempenho escolar, e de forma generalizada, afirmam a existência da relação.

Na tentativa de verificar que fatores, representados por indicadores, influenciam o desempenho de estudantes das Séries Iniciais do Ensino Fundamental nas avaliações em larga escala dos municípios de pequeno porte da Região Sul do Brasil, analisou-se o indicador Incidência de Pobreza, disponibilizado pelo IBGE para todos os municípios do país em relação ao rendimento escolar mensurado pelo IDEB.

\section{POBREZA E RENDIMENTO ESCOLAR}

Em se tratando da pobreza, diversos conceitos e determinantes compõem o cenário dos debates acadêmicos e políticos que buscam consenso. $O$ entendimento sobre qual a linha divisória entre pobres e não pobres, entretanto, é muito tênue e com diferentes métodos de definição.

A definição de pobreza está relacionada à privação, cuja natureza pode ser absoluta, relativa ou subjetiva, dadas as carências de ordem material, cultural e social, em face à disponibilidade e ao usufruto de recursos de um indivíduo ou de um grupo social (Kageyama; Hollfmann, 2006). Nessa perspectiva, inicialmente, pode-se definir a pobreza por meio de três categorias: a pobreza absoluta, a relativa e a subjetiva, associadas, respectivamente, à não satisfação de um mínimo definido socialmente; à privação de bens e serviços, considerados comuns numa sociedade; e ao sentimento de impotência.

O conceito de pobreza absoluta leva em consideração as necessidades básicas e refere-se à ausência ou à insuficiência de renda para a satisfação de mínimos sociais necessários à sobrevivência física. Dessa forma, são classificados como pobres todos os indivíduos cujo baixo padrão de renda impossibilita o acesso às necessidades básicas de manutenção e sobrevivência física. Vale dizer que 
essa concepção, apesar de conferir objetividade à noção de pobreza, pode ser considerada parcial, já que enfoca apenas a dimensão monetarista (econômica) e subestima outros aspectos relevantes à manutenção e à reprodução da vida, por não considerar variáveis não econômicas, relacionando pobreza estritamente à renda (Rocha, 2005).

Estudos do Banco Mundial desenvolvidos por Elbers, Lanjouw e Lanjouw (2003) adotam procedimentos estatísticos que combinam informações detalhadas, coletadas em pesquisas domiciliares amostrais, com a ampla cobertura geográfica do Censo Demográfico. Com base nesse método, o IBGE construiu o Mapa da Pobreza, utilizando as informações da Pesquisa de Orçamentos Familiares (POF 2002 -2003). A proposta foi, com base no gasto per capita domiciliar, imputar informações equivalentes para os domicílios do Censo Demográfico, no sentido de propiciar a estimação de medidas de pobreza e desigualdade. A utilização deste método permitiu calcular a pobreza e a desigualdade em pequenas áreas, bem como avaliar a precisão das estimativas.

Embora haja o reconhecimento de que a pobreza é um fenômeno complexo, caracterizado por carências de diversos tipos, observa-se que, de modo geral e recorrente, a renda é utilizada como determinante do bem-estar - análise unidimensional. Portanto, a avaliação de sua dimensão é baseada inicialmente na insuficiência de renda para o usufruto do conjunto de bens e serviços correspondentes ao necessário para se viver em sociedade.

O mais elementar, e também o mais amplamente empregado, meio de avaliar a dimensão da pobreza, em qualquer sociedade, é o de obter uma simples contagem dos pobres. Os pobres são aqueles cujos rendimentos ficam aquém do estipulado numa linha da pobreza. A medida mais comum da pobreza é o Índice de Incidência da Pobreza, o qual constitui a proporção de pobres na população total.

Um forte argumento a favor do uso do Índice de Incidência da Pobreza é que ele cumpre o que se pode chamar um "Princípio da Probabilidade", que é a exigência de que uma medida da pobreza deva transmitir algumas informações sobre a probabilidade de encontrar uma pessoa pobre em qualquer sociedade.

A pobreza no Brasil tem um forte componente regional: sua incidência é mais elevada no Norte e no Nordeste, independente dos indicadores utilizados, reduzindo-se em direção ao Sul. 
Para o conjunto do país, embora a proporção de pobres se mantenha ainda mais elevada em áreas rurais (25\%), os pobres urbanos já são largamente a maioria, correspondendo a $75 \%$ dos pobres brasileiros.

Aqui será utilizada apenas a proporção de pobres na população total, a partir do indicador de pobreza, caracterizado pela insuficiência de renda (ROCHA, 1998). O objetivo é analisar as possíveis relações entre o valor médio do IDEB, e a Incidência de Pobreza nos municípios de pequeno porte da Região Sul do Brasil.

A figura 01 traz os resultados do cruzamento do Valor médio do IDEB e da Incidência de Pobreza nos municípios de pequeno porte da Região Sul divididos por Estado.

Figura 01: Relação entre Valor médio do IDEB e Incidência de Pobreza nos municípios de pequeno porte nos três estado da Região Sul.
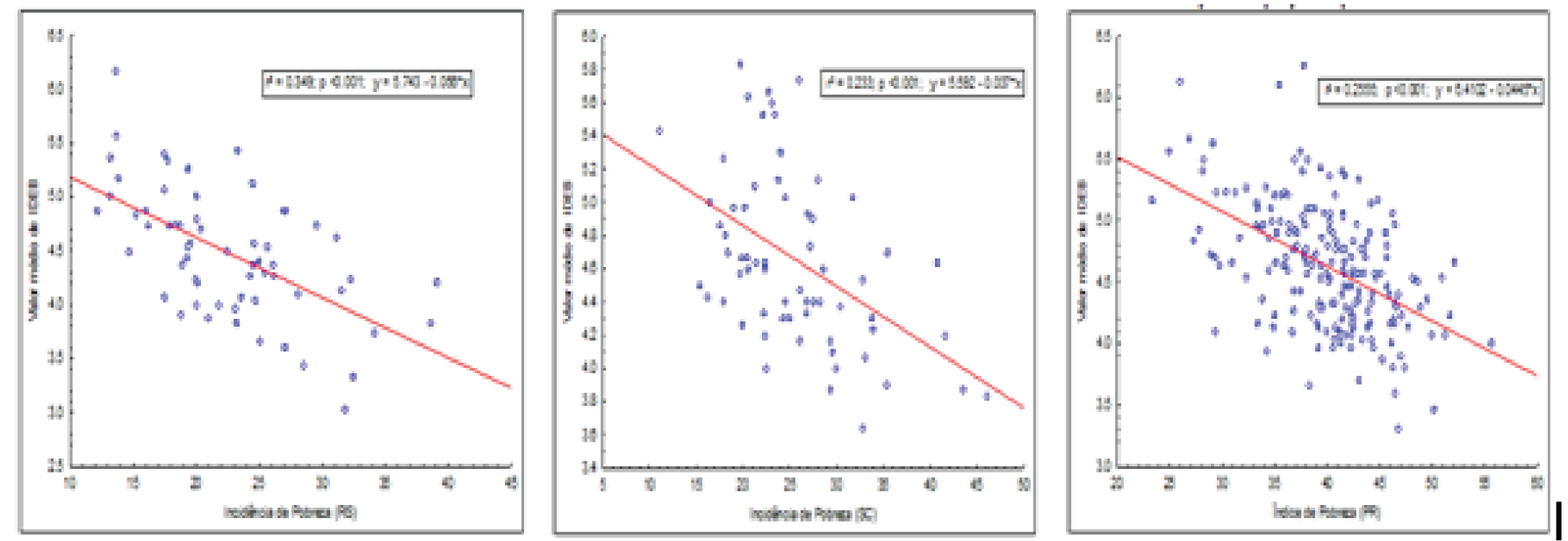

Como método de análise, utilizou-se a regressão linear entre as variáveis Incidência de Pobreza e Valor médio de IDEB para municípios com menos de 10 mil habitantes e que apresentam ambas as variáveis. Os valores indicam que há uma relação inversamente proporcional, demonstrando que, conforme aumentam os valores da Incidência de Pobreza, diminuem os valores médios de IDEB. A relação é significativa. $O$ índice que mede a relação apresenta valor médio, demonstrando que se trata de uma associação considerável.

A diferença entre o índice que mede a relação $\left(r^{2}\right)$ é significativa entre o Rio Grande do Sul e Santa Catarina. O valor ultrapassa os $10 \%$ de casos que se ajustam à reta do gráfico, significando que a relação para o estado do Rio Grande do Sul é mais confiável do que a de Santa Catarina.

Os gráficos dos três estados apresentam resultados semelhantes. A relação entre as duas variáveis consideradas é significativa, com uma associação considerável. Os valores de $r^{2}$ são significativos, ainda que diferentes entre os 
estados. Trata-se da medida estatística que define a porcentagem da variável dependente (valor médio do IDEB), que pode ser identificada pela equação de regressão linear e possibilita avaliar se os valores de x (variável independente Incidência de Pobreza) permitem, ou não, proceder a uma boa estimativa de y (variável dependente média do IDEB).

Rio Grande do Sul se destaca com o maior índice de relação $r^{2}=0,349$, ou seja, 34,9\% dos valores das variáveis se ajustam perfeitamente à reta do gráfico, demonstrando uma relação significativa. O Paraná apresenta um valor mais baixo, sendo $r^{2}=0,255$ ou $25,5 \%$ dos casos se ajustam perfeitamente à reta e Santa Catarina tem os valores de $r^{2}=0,233$, ou 23,3\%.

Os valores de p são iguais para os três estados. O indicador pé menor que 0,001. Este valor indica que a hipótese inicial $(\mathrm{Ho})$ é verdadeira, pois a probabilidade de negar a hipótese de que o Valor médio do IDEB é diretamente relacionável com a Incidência de Pobreza, nos municípios de pequeno porte da Região Sul do Brasil, é praticamente nula.

Esses dados reforçam as conclusões de outros estudos (Soares, 2004; Alves 2008; Coelho, 2008, entre outros) que afirmam que as condições sociais incidem no rendimento escolar. Quanto pior for a distribuição de renda e quanto maior for a Incidência de Pobreza, piores são os resultados das avaliações padronizados que resultam no IDEB.

Quando se analisa a Região Sul desconsiderando a divisão por estados, o resultado demonstra que há uma relação inversamente proporcional, que, conforme aumentam os valores da Incidência de Pobreza, diminuem os valores médios de IDEB e que esta relação é significativa, assim como na análise anterior, entretanto o índice que mede a relação apresenta um valor baixo, demonstrando que se trata de uma associação fraca para esta amplitude.

Este resultado coloca diante de uma problemática significativa em torno das avaliações em larga escala reduzidas nos valores do IDEB, pois quando se analisa por regiões menores, como as unidades federativas em separado, há valores mais confiáveis; já quando se ampliam o espaço e, consequentemente, o número de casos, obtêm-se valores mais fracos em torno da associação. Isso pode significar que os valores do IDEB como indicador de qualidade da educação para o país ou região não é confiável, já que para amplitudes maiores os indicadores perdem precisão. 
Noâmbito geral, considerando os três estados, os efeitos dos valores daIncidência de Pobreza, apesar de apresentarem associação positiva, encontram-se diluídos. Isso se deve à composição dessa variável, que é dissociada em seus componentes menores, como a renda familiar mensal nominal per capita, por exemplo.

A diferença de resultados aponta para um cuidado que se deve ter ao analisar dados de regiões com realidades diferentes. Teoricamente, tem-se o mesmo conjunto de dados; portanto, dever-se-ia ter o mesmo resultado. Ao se ampliar o espaço da análise, dilui-se a relação. Então, as diferenças entre os estados desaparecem, formando uma única massa, cujos resultados apresentam uma relação menos confiável.

Ao se generalizar essa conclusão, em hipótese, tem-se a fragilidade das afirmações com dados estatísticos para grandes regiões, englobando diferentes realidades. Se se percebe que há diferença entre a relação das variáveis por estado e no total da Região Sul para os valores dos pequenos municípios, podese deduzir que as variações sejam ainda mais significativas em estudos que considerem o total dos valores, independentemente da faixa populacional ou de qualquer outro critério de diferenciação das realidades.

Portanto, estudos estatísticos que buscam comparar realidades mais próximas tendem a ser mais eficientes do que estudos com realidades muito diferentes. Pode-se reduzir esse fato ao princípio básico da comparabilidade, que é comparar aquilo que é comparável.

\section{CONSIDERAÇÕES E APONTAMENTOS}

O objetivo deste estudo foi analisar se há relação entre a Incidência de Pobreza e o IDEB nos municípios de pequeno porte da Região Sul do Brasil, buscando complementar as análises sobre as influências de indicadores sociais sobre o desempenho escolar auferido pelas avaliações em larga escala.

A metodologia de análise desenvolvida para esta pesquisa revelou a fragilidade das modelagens estatísticas para grandes espaços, devido à variação das condições socioeconômicas e populacionais da realidade brasileira. A Região Sul, composta por três estados, quando comparados com o restante do país, é considerada equilibrada em termos de qualidade de vida, renda e condições sociais, entretanto demonstrou diferenças significativas nos resultados das 
análises. Enquanto a associação entre as variáveis IDEB e Incidência de Pobreza por unidade federativa foi significativa e forte, o resultado para a Região Sul demonstrou ser fraco. Em hipótese dever-se-ia ter o mesmo resultado, já que a análise da Região Sul foi efetuada a partir da soma dos dados das três unidades federativas. No entanto isso não ocorreu, devido à diluição das especificidades no todo, gerando uma falsa homogeneidade.

Mesmo considerando a fragilidade dos modelos estatísticos e da própria produção de dados, há apontamentos confiáveis da existência de relação entre IDEB e Incidência de Pobreza. A pesquisa revelou que quanto maior a porcentagem de pobres no município, menor foi a variação positiva do IDEB. Isso reafirma os resultados de pesquisas citadas anteriormente que já indicavam tal relação. Entretanto, não apontavam para a diferença entre espaços menores e maiores nos modelos estatísticos. Esta é uma das contribuições desta pesquisa, que cumpre seu papel inicial ao significar os indicadores para o uso na administração. Nestes termos, só será possível melhorar a qualidade da educação melhorando a qualidade de vida da população. Quando se afirma que há relação entre a Incidência de Pobreza e o rendimento escolar, tanto demonstrado por esta pesquisa quanto por inúmeros outros estudos com metodologias diferentes, está-se apontando para um problema que extrapola a sala de aula e os muros das escolas, que, por vezes, não são resolvidos com ações e/ou políticas educacionais isoladas e descontextualizadas.

O fato do pouco uso dos dados estatísticos produzidos por instituições governamentais como o IBGE e INEP pelos gestores pode ser explicado pelo pouco conhecimento e domínio dos dados, mas também pela falta de estudos na área que consigam integrar as informações, dando maior sentido aos números, aproximando setores, órgãos ou secretarias com funções distintas, mas que repercutem diretamente no desempenho escolar.

A pobreza não é uma questão apenas de falta de renda, mas também de acesso a bens culturais que possibilitam maior desenvolvimento intelectual. Neste aspecto, o rendimento escolar depende diretamente das ações governamentais de diminuição da pobreza. Ações que interferem não só nas escolas, mas na saúde, alimentação, segurança e lazer.

Políticas públicas de regulação, avaliação e responsabilização são validas quando as condições básicas de vida são respeitadas. A pesquisa indica que, 
quando há menos pobres, melhor é o rendimento das crianças nas avaliações em larga escala. Quando há mais pobres, tem-se menor desempenho. Só isso já bastaria para que se tivessem políticas públicas voltadas para a diminuição da pobreza como medida de garantia da melhoria da qualidade da educação.

\section{REFERÊNCIAS BIBLIOGRÁFICAS}

ALVES, F. Qualidade na educação fundamental pública nas capitais brasileiras: tendências, contextos e desafios. Belo Horizonte: Argvmentvm, 2008.

ANDRADE, J. M. de \& Laros, J. A. Fatores associados ao desempenho escolar: estudo multinível com dados do SAEB/2001. Psicologia: Teoria e Pesquisa, Vol. 23, n. 1, p. 33-42, Jan-Mar 2007.

BARBOSA, M. E. F. \& FERNANDES, C. A escola faz diferença? Uma investigação dos feitos da escola na proficiência em matemática dos alunos da 4a série. Em C. Franco (Org.), Avaliação, ciclos e promoção na educação. Porto Alegre: ArtMed, 2001, 155-172.

COELHO, M. I. de M. Vinte anos de avaliação da educação básica no Brasil: aprendizagens e desafios. Ensaio: avaliação e políticas públicas em educação. RJ, v. 16, n 59, p. 229-258, abr/ jun. 2008.

ELBERS, C.; LANJOUW, J. O.; LANJOUW, P.; Micro--Level Estimation of Poverty and Inequality. Econometrica, Econometric Society, vol. 71(1), p. 355-364, Janeiro. 2003.

GANZEBOOM, H. B. G.; DE GRAAF, P. M.; TREIMAN, D. J. A standard international socio-economic index of occupational status. Social Science Research. Vol. 21, 1992, p 01-56.

KAGEYAMA, Â.; HOFFMANN, R. Pobreza no Brasil: uma perspectiva multidimensional. Revista Economia e Sociedade. Campinas: v.15, n.1(26), p. 79-112, jan-jun, 2006.

ROCHA, S. Pobreza no Brasil: afinal, de que se trata? Rio de Janeiro: 2. ed. Editora FGV, 2005.

ROCHA, S. Pobreza no Brasil. Principais tendências da espacialização. Economia e Sociedade. Campinas, p. 193-211, 1998.

SOARES, M. Alfabetização no Brasil - O Estado do conhecimento. Brasília: INEP/ MEC, 2004.

SOARES, J. F.; CESAR, C. C. \& Mambrini, J. Determinantes de desempenho dos alunos do ensino básico brasileiro: evidências do SAEB de 1997. IN: C. Franco (Org.), Avaliação, ciclos e promoção na educação. Porto Alegre: Artmed, 2001, p. 121-154. 
SOUZA, P. R. Avaliação a Serviço da Qualidade Educativa. 2004. Disponível em www. Reescrevendoaeducação.com.br/2006/pages.php?recid=44 - 44.

Artigo recebido em: 26/11/2015 Aprovado em: 02/05/2016

Endereço para correspondência:

Valdecir Soligo. Rua Belas Artes, 307, Bairro Universitário, Casvavel, PR, CEP: 85819-180. E-mail: valdecir_soligo@yahoo.com.br 